Mathematical Models and Methods in Applied Sciences

(C) World Scientific Publishing Company

\title{
AN ENERGETICALLY CONSISTENT VISCOUS SEDIMENTATION MODEL
}

\author{
JEAN DE DIEU ZABSONRÉ \\ LAMA, UMR 5127, Université de Savoie, \\ 73376 Le-Bourget-du-Lac Cedex, France. \\ wend-woaga.zabsonre@univ-savoie.fr \\ Université de Ouagadougou, UFR/SEA, LAME, \\ 03 BP 7021 Ouagadougou 03, Burkina Faso. \\ jzabsonre@univ-ouaga.bf \\ Université Polytechnique de Bobo-Dioulasso, ISEA, \\ 01 BP 1091 Bobo 01, Burkina Faso.
}

\section{CARINE LUCAS}

Université de Grenoble and INRIA, LJK, UMR 5224, 51 Rue des Mathématiques, B.P. 53, 38041 Grenoble, France.

carine.lucas@imag.fr

ENRIQUE FERNÁNDEZ-NIETO

Departamento de Matemática Aplicada I, E. T. S. Arquitectura, Universidad de Sevilla Avda Reina Mercedes N.2, 41012 Sevilla, Spain.

edofer@us.es

Received (Day Month Year)

Revised (Day Month Year)

Communicated by (xxxxxxxxx)

\begin{abstract}
In this paper we consider a two dimensional viscous sedimentation model which is a viscous Shallow-Water system coupled with a diffusive equation that describes the evolution of the bottom. For this model, we prove the stability of weak solutions for periodic domains and give some numerical experiments. We also discuss around various discharge quantity choices.
\end{abstract}

Keywords: Sedimentation ; shallow water ; viscous models ; energetic consistency ; stability.

AMS Subject Classification: 35Q30

\section{Introduction.}

Phenomena related to sediment transport have a huge interest as they affect human life and earthly morphology in a determinant way. Indeed, the geomorphological evolution of the rivers under the effect of the hydrodynamic transport of the sediments which compose their beds constitutes a fundamental problem for the management 
2 J. D. D. Zabsonré, C. Lucas E豸 E. Fernández-Nieto

of the rivers, the mastery of the environmental risks and the fight against the floods. The analysis of sediment transport is then important to predict and prevent natural disasters.

For this purpose, many physical and mathematical models are proposed in the literature in order to predict the bed evolution and the changes in water regime when such unsteady flows occur. Physical experiments are particularly used to calculate local scouring phenomena, such as the local erosion around the bridge pier or the scour hole due to a jet issued from a underflow gate. However, when problems with large space or times scales have to be solved, a mathematical model is generally required.

Among the mathematical models, the most often used is based on the Saint-VenantExner equations. This model, studied numerically in Ref. 10 and Ref. 19 for example, couples an hydrodynamic Saint-Venant (Shallow-Water) system to a morphodynamic bed-load transport sediment equation (similar to the one introduced in Ref. 21) as follows:

$$
\begin{aligned}
& \partial_{t} h+\operatorname{div} q=0, \\
& \partial_{t} q+\operatorname{div}\left(\frac{q \otimes q}{h}\right)+\frac{1}{F r^{2}} h \nabla\left(h+z_{b}\right)=0,
\end{aligned}
$$

and

$$
\partial_{t} z_{b}+\xi \operatorname{div}\left(q_{b}(h, q)\right)=0
$$

where $\mathrm{Fr}$ is the Froude number (ratio between the characteristic velocity of the fluid and the square root of the product of the gravity and the characteristic height), $z_{b}$ is the movable bed thickness, $\xi=1 /\left(1-\psi_{0}\right)$ with $\psi_{0}$ the porosity of the sediment layer and $q_{b}$ denotes the solid transport flux or sediment discharge. It depends on the height $h$ of the fluid and the water discharge $q=h u$, where $u$ is the velocity (see Figure 1).

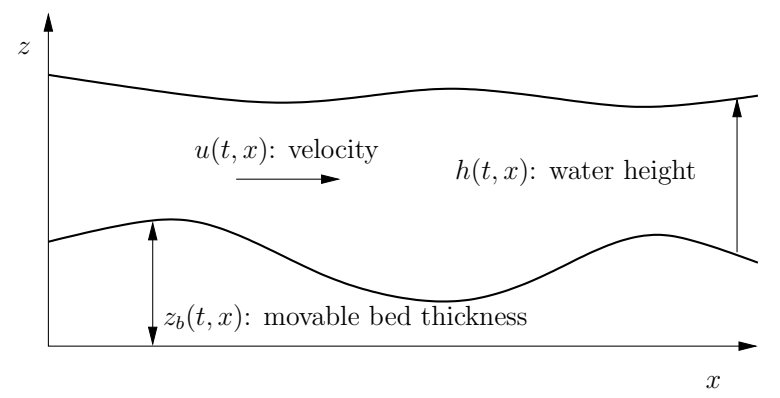

Fig. 1. Sediment and water heights

For the solid transport flux $q_{b}$, there exists several formulas in the literature: the Grass equation, ${ }^{11}$ the Meyer-Peter and Muller equation, ${ }^{16}$ or the formulas of 
Fernández Luque and Van Beek, of Van Rijn, or of Nielsen ${ }^{10,19}$. All of them are obtained using empirical methods. The most basic sediment model is the Grass equation, where the sediment movement begins at the same time as the fluid motion. In this case, the solid transport flux is given by

$$
q_{b}(h, q)=A_{g} \frac{q}{h}\left|\frac{q}{h}\right|^{m_{g}}=A_{g}|u|^{m_{g}} u, \quad 0 \leq m_{g} \leq 3,
$$

where the constant $A_{g}$ includes the effects due to grain size and kinematic viscosity.

However, System (1.1)-(1.3) does not take the viscosity into account. In the viscous case, we have to consider the viscous version of the Shallow-Water system. Several choices have been considered in the literature for the viscous term ${ }^{14}$ : in Ref. 17, the author chose the laplacian and obtained an existence result, but this system is not energetically consistent. In Ref. 1 and 2, the viscous terms are $\operatorname{div}(h \nabla u)$ or $\operatorname{div}(h D(u))$, which gives an energetically consistent system. In this case, the authors proved the existence of global weak solutions. The key point in those papers is to show that the structure of the diffusion term provides some extra regularity for the density thanks to a new mathematical entropy inequality named BD entropy. But note that the stability result is obtained using drag and turbulence terms or capillarity. Recently, keeping this choice of viscous terms but without any additional regularizing terms, MELLET and VASSEUR ${ }^{15}$ proved the stability of a class of barotropic compressible Navier-Stokes equations, which includes the case of the viscous energetically consistent Shallow-Water system. This paper also uses the new BD entropy with an extra key point which gives bounds on $h u^{2}$ in a better space than $L^{\infty}\left(0, T ; L^{1}(\Omega)\right)$, thanks to new multipliers, namely $|u|^{k} u$ and $u+u \log \left(1+|u|^{2}\right)$.

Let us recall now the existing results on the viscous sedimentation models, that are a viscous Shallow-Water system coupled with an evolution equation for the bottom. A recent work ${ }^{21}$ has been done on a model that couples the Shallow-Water system studied by OrengA

$$
\begin{aligned}
& \partial_{t} h+\operatorname{div}(h u)=0 \\
& \partial_{t} u+u \cdot \nabla u+\frac{1}{F r^{2}} \nabla\left(h+z_{b}\right)-\Delta u=f,
\end{aligned}
$$

with a Grass equation (1.4) satisfying

$$
\xi q_{b}=h u .
$$

As the authors assume small variations of the free surface around a fixed level $(z=c s t)$, they replace $h$ by $-z_{b}$ in (1.7). Then they follow the lines given by Ref. 17: thanks to a Brower fixed point on the finite dimensional problem, they get global existence results assuming the data to be small enough.

In this article, we propose a new viscous sedimentation model, stable and energetically consistent. It consists in coupling a viscous Shallow-Water system with a sediment diffusive equation in a bounded domain with periodic boundary conditions, that is $\Omega=T^{2}$. More precisely, if we denote by $\nu$ the non-dimensional 
viscosity ( $\nu=2 / R e$, where $R e$ is the Reynolds number) and $A$ a positive constant, we consider the following system

$$
\begin{aligned}
& \partial_{t} h+\operatorname{div}(h u)=0, \\
& \partial_{t}(h u)+\operatorname{div}(h u \otimes u)+\frac{h \nabla\left(h+z_{b}\right)}{F r^{2}}-\nu \operatorname{div}(h D(u))=0, \\
& \partial_{t} z_{b}+A \operatorname{div}\left(h|u|^{k} u\right)-\frac{\nu}{2} \Delta z_{b}=0,
\end{aligned}
$$

with the initial conditions

$$
\left.h\right|_{t=0}=h_{0} \geq 0,\left.\quad z_{b}\right|_{t=0}=z_{b_{0}},\left.\quad h u\right|_{t=0}=m_{0},
$$

where $D(u)$ is the symmetric part of the gradient, $D(u)=\left(\nabla u+{ }^{t} \nabla u\right) / 2, \operatorname{Fr}>0$ denotes the Froude number, $k$ is a positive real number satisfying $0<k<1 / 2$. The initial data are taken in such a way that

$$
\begin{gathered}
h_{0} \in L^{2}(\Omega), \quad z_{b_{0}} \in L^{2}(\Omega), \\
\frac{\left|q_{0}\right|^{2}}{h_{0}} \in L^{1}(\Omega), \quad \nabla \sqrt{h_{0}} \in\left(L^{2}(\Omega)\right)^{2} .
\end{gathered}
$$

After stating the main results in Section 2, we establish, in Section 3 some energy and entropy relations that give us a priori estimates. These estimates are then used in Section 4 to prove the announced theorem. We also propose numerical experiments on this new model in Section 6, and conclude, in Section 5, with two other models of sedimentation, inspired by the works mentioned above. More precisely, we first study the model considered in Ref. 21 but with the viscous Shallow-Water system (1.8)-(1.9) and, in a second part, we introduce one of the multipliers used by Mellet and Vasseur in the sediment equation.

\section{Main results.}

In this part, we first recall the definition that will be used in the following. We then give the main theorem of this paper that will be proved in Section 4 .

\subsection{Notion of weak solutions.}

We shall say $\left(h, u, z_{b}\right)$ is a weak solution of (1.8)-(1.10) on $(0, T) \times \Omega$ with initial conditions (1.11) if

- System (1.8)-(1.10) holds in $\left(\mathcal{D}^{\prime}((0, T) \times \Omega)\right)^{4}$,

- Eq. (1.11) (on initial conditions) holds in $\mathcal{D}^{\prime}(\Omega)$ with $h \geq 0$ a.e.,

- The Energy inequality (3.1) is satisfied for a.e. non negative $t$ and the following regularity properties are satisfied

$$
\begin{array}{ll}
\sqrt{h} u \in L^{\infty}\left(0, T ;\left(L^{2}(\Omega)\right)^{2}\right), & \sqrt{h} \nabla u \in L^{2}\left(0, T ;\left(L^{2}(\Omega)\right)^{4}\right), \\
h^{1 /(k+2)} u \in L^{\infty}\left(0, T ;\left(L^{k+2}(\Omega)\right)^{2}\right), & h+z_{b} \in L^{\infty}\left(0, T ; L^{2}(\Omega)\right), \\
\nabla h+\nabla z_{b} \in L^{2}\left(0, T ;\left(L^{2}(\Omega)\right)^{2}\right), & \nabla \sqrt{h} \in L^{\infty}\left(0, T ;\left(L^{2}(\Omega)\right)^{2}\right), \\
h^{1 / k} D(u)^{2 / k} u \in L^{k}\left(0, T ;\left(L^{k}(\Omega)\right)^{2}\right) . &
\end{array}
$$


An energetically consistent viscous sedimentation model 5

\subsection{Main theorem.}

The main result of this paper is the following:

Theorem 2.1. Let $\left(h_{n}, u_{n}, z_{b_{n}}\right)$ be a sequence of weak solutions of (1.8)-(1.10) satisfying entropy inequalities (3.1), (3.4), with initial data

$$
h_{\left.n\right|_{t=0}}=h_{0}^{n}(x), h_{n} u_{\left.n\right|_{t=0}}=q_{0}^{n}(x) \text { and } z_{\left.b_{n}\right|_{t=0}}=z_{b_{0}}^{n}(x),
$$

where $h_{0}^{n}, z_{b_{0}}^{n}$ and $u_{0}^{n}$ verify

$$
h_{0}^{n} \geq 0, \quad h_{0}^{n} \rightarrow h_{0} \text { in } L^{1}(\Omega), \quad z_{b_{0}}^{n} \rightarrow z_{b_{0}} \text { in } L^{1}(\Omega), \quad q_{0}^{n} \rightarrow q_{0} \text { in } L^{1}(\Omega),
$$

and satisfy the following bounds:

$$
\begin{gathered}
\int_{\Omega} h_{0}^{n} \frac{\left|u_{0}^{n}\right|^{2}}{2}+\frac{\left|h_{0}^{n}+z_{b_{0}}^{n}\right|^{2}}{2}+h_{0}^{n} \frac{\left|u_{0}^{n}\right|^{k+2}}{k+2}<C, \\
\int_{\Omega}\left|\nabla \sqrt{h_{0}^{n}}\right|^{2}<C \text { and } \int_{\Omega}\left|h_{0}^{n}\right|<C .
\end{gathered}
$$

Then, up to a subsequence, $\left(h_{n}, u_{n}, z_{b_{n}}\right)$ converges strongly to a weak solution of (1.8)-(1.10) satisfying entropy inequalities (3.1) and (3.4).

\section{Energy estimates and BD entropy.}

In this section, we give some energy and entropy inequalities. These relations will be used in Section 4 where we prove Theorem 2.1. But let us first recall the energy inequality in the inviscid case, for System (1.1)-(1.3).

\subsection{The case without viscosity.}

Lemma 3.1. Let $\left(h, u, z_{b}\right)$ be a smooth solution of the system

$$
\begin{aligned}
& \partial_{t} h+\operatorname{div} q=0, \\
& \partial_{t} q+\operatorname{div}(h u \otimes u)+\frac{1}{F r^{2}} h \nabla\left(h+z_{b}\right)=0, \\
& \partial_{t} z_{b}+A \operatorname{div}\left(h|u|^{k} u\right)=0 .
\end{aligned}
$$

Then the following identity holds:

$$
\frac{1}{2} \frac{d}{d t} \int_{\Omega} h|u|^{2}+\frac{A}{k+2} \frac{d}{d t} \int_{\Omega} h|u|^{k+2}+\frac{1}{2 F r^{2}} \frac{d}{d t} \int_{\Omega}\left|h+z_{b}\right|^{2}=0 .
$$

The proof of this lemma will be included in the viscous case.

\subsection{The viscous case.}

From now on, we consider the viscous system (1.8)-(1.10). 
Proposition 3.1. Let $\left(h, u, z_{b}\right)$ be a smooth solution of (1.8)-(1.10). Then the following energy inequality holds:

$$
\begin{aligned}
\frac{1}{2} \frac{d}{d t} \int_{\Omega} h|u|^{2}+\frac{1}{2 F r^{2}} \frac{d}{d t} \int_{\Omega}\left|z_{b}+h\right|^{2} & +\frac{A}{k+2} \frac{d}{d t} \int_{\Omega} h|u|^{k+2} \\
+\frac{\nu}{2 F r^{2}} \int_{\Omega} \nabla h \cdot \nabla z_{b}+ & \frac{\nu}{2 F r^{2}} \int_{\Omega}\left|\nabla z_{b}\right|^{2}+\frac{\nu}{4} \int_{\Omega} h\left|\nabla u+{ }^{t} \nabla u\right|^{2} \\
& +\frac{1-2 k}{4} A \nu \int_{\Omega} h\left|\nabla u+{ }^{t} \nabla u\right|^{2}|u|^{k} \leq 0 .
\end{aligned}
$$

Proof. We multiply Eq. (1.9) by $u$, and integrate on $\Omega$. This gives, using (1.8):

$$
\int_{\Omega} h \partial_{t} u \cdot u+\int_{\Omega}(h u \cdot \nabla) u \cdot u+\frac{1}{F r^{2}} \int_{\Omega} h \nabla\left(h+z_{b}\right) \cdot u-\nu \int_{\Omega} \operatorname{div}(h D(u)) \cdot u=0 .
$$

Now let us simplify each term:

- $\int_{\Omega} h \partial_{t} u \cdot u+\int_{\Omega}(h u \cdot \nabla) u \cdot u=\frac{1}{2} \frac{d}{d t} \int_{\Omega} h|u|^{2}$,

- $\int_{\Omega} h \nabla\left(h+z_{b}\right) \cdot u=\int_{\Omega}\left(h+z_{b}\right) \partial_{t} h=\frac{1}{2} \frac{d}{d t} \int_{\Omega} h^{2}+\int_{\Omega} z_{b} \partial_{t} h$,

- $\int_{\Omega} \operatorname{div}(h D(u)) \cdot u=-\int_{\Omega} h D(u): \nabla u=-\frac{1}{4} \int_{\Omega} h\left|\nabla u+{ }^{t} \nabla u\right|^{2}$.

Substituting all these terms, we get:

$$
\frac{1}{2} \frac{d}{d t} \int_{\Omega} h|u|^{2}+\frac{1}{2 F r^{2}} \frac{d}{d t} \int_{\Omega} h^{2}+\frac{1}{F r^{2}} \int_{\Omega} z_{b} \partial_{t} h+\frac{\nu}{4} \int_{\Omega} h\left|\nabla u+{ }^{t} \nabla u\right|^{2}=0 .
$$

Contrary to the study of the classical Shallow Water system, we cannot make any assumption on the regularity of the bottom $z_{b}$ : we have to use the energy relations to get such properties. That is the reason why we are led to carry on the calculation. We multiply Eq. (1.9) by $|u|^{k} u$ and we integrate on $\Omega$ :

$$
\begin{aligned}
\int_{\Omega} h \partial_{t} u \cdot|u|^{k} u+\int_{\Omega}(h u \cdot \nabla) u \cdot|u|^{k} u+\frac{1}{F r^{2}} \int_{\Omega} & h \nabla\left(h+z_{b}\right) \cdot|u|^{k} u \\
& -\nu \int_{\Omega} \operatorname{div}(h D(u)) \cdot|u|^{k} u=0 .
\end{aligned}
$$

Here again, we study separately each term:

$$
\begin{aligned}
& -\int_{\Omega} h \partial_{t} u \cdot|u|^{k} u+\int_{\Omega}(h u \cdot \nabla) u \cdot|u|^{k} u=\frac{1}{k+2} \frac{d}{d t} \int_{\Omega} h|u|^{k+2}, \\
& \text { - } \frac{1}{F r^{2}} \int_{\Omega} h \nabla\left(h+z_{b}\right) \cdot|u|^{k} u=-\frac{1}{F r^{2}} \int_{\Omega}\left(h+z_{b}\right) \operatorname{div}\left(h|u|^{k} u\right) .
\end{aligned}
$$


An energetically consistent viscous sedimentation model 7

Then we use Eq. (1.10) to write:

$$
\begin{gathered}
\begin{array}{c}
\frac{1}{F r^{2}} \int_{\Omega} h \nabla\left(h+z_{b}\right) \cdot|u|^{k} u \\
=-\frac{\nu}{2 A F r^{2}} \int_{\Omega}\left(h+z_{b}\right) \Delta z_{b}+\frac{1}{A F r^{2}} \int_{\Omega}\left(h+z_{b}\right) \partial_{t} z_{b} \\
=\frac{\nu}{2 A F r^{2}} \int_{\Omega} \nabla h \cdot \nabla z_{b}+\frac{\nu}{2 A F r^{2}} \int_{\Omega}\left|\nabla z_{b}\right|^{2}+\frac{1}{A F r^{2}} \int_{\Omega} h \partial_{t} z_{b} \\
\quad+\frac{1}{2 A F r^{2}} \frac{d}{d t} \int_{\Omega} z_{b}{ }^{2}, \\
\text { - } \int_{\Omega} \operatorname{div}(h D(u)) \cdot|u|^{k} u=-\frac{1}{4} \int_{\Omega} h\left|\nabla u+{ }^{t} \nabla u\right|^{2}|u|^{k} \\
\text { and }\left.\left|\int_{\Omega}(h D(u) u \cdot \nabla) u \cdot u\right| u\right|_{\Omega} ^{k-2}(h D(u) u \cdot \nabla) u \cdot u|u|^{k-2},
\end{array}
\end{gathered}
$$

Gathering all these results, we are led to:

$$
\begin{aligned}
\frac{1}{k+2} \frac{d}{d t} \int_{\Omega} h|u|^{k+2} & +\frac{\nu}{2 A F r^{2}} \int_{\Omega} \nabla h \cdot \nabla z_{b}+\frac{\nu}{2 A F r^{2}} \int_{\Omega}\left|\nabla z_{b}\right|^{2}+\frac{1}{A F r^{2}} \int_{\Omega} h \partial_{t} z_{b} \\
+ & \frac{1}{2 A F r^{2}} \frac{d}{d t} \int_{\Omega} z_{b}{ }^{2}+\frac{1-2 k}{4} \nu \int_{\Omega} h\left|\nabla u+{ }^{t} \nabla u\right|^{2}|u|^{k} \leq 0 .
\end{aligned}
$$

Now we multiply Eq. (3.3) by $A$ and we add Equality (3.2): we find the proclaimed inequality.

However, we still do not know the sign of the integral of $\nabla h \cdot \nabla z_{b}$. To get more information, we study the BD entropy.

Proposition 3.2. For $\left(h, u, z_{b}\right)$ solution of the model (1.8)-(1.10), we show the following relation:

$$
\begin{aligned}
\frac{1}{2} \frac{d}{d t} \int_{\Omega} h \mid u & +\left.\nu \nabla \log h\right|^{2}+\frac{1}{F r^{2}} \frac{d}{d t} \int_{\Omega}\left|z_{b}+h\right|^{2}+\frac{2 A}{k+2} \frac{d}{d t} \int_{\Omega} h|u|^{k+2} \\
+ & \frac{1}{2} \frac{d}{d t} \int_{\Omega} h|u|^{2}+\frac{\nu}{F r^{2}} \int_{\Omega}\left|\nabla\left(h+z_{b}\right)\right|^{2}+\frac{\nu}{4} \int_{\Omega} h\left|\nabla u+{ }^{t} \nabla u\right|^{2} \\
& +\frac{\nu}{4} \int_{\Omega} h\left|\nabla u-{ }^{t} \nabla u\right|^{2}+\frac{1-2 k}{4} A \nu \int_{\Omega} h\left|\nabla u+{ }^{t} \nabla u\right|^{2}|u|^{k} \leq 0 .
\end{aligned}
$$

The proof relies on the following lemma:

Lemma 3.2. If $\left(h, u, z_{b}\right)$ is a solution of the model (1.8)-(1.10), we have the equality:

$$
\begin{aligned}
\frac{\nu^{2}}{2} \frac{d}{d t} \int_{\Omega} h|\nabla \log h|^{2} & +\frac{\nu}{F r^{2}} \int_{\Omega}|\nabla h|^{2} \\
& =-\nu \frac{d}{d t} \int_{\Omega} u \cdot \nabla h+\nu \int_{\Omega} h \nabla u:{ }^{t} \nabla u-\frac{\nu}{F r^{2}} \int_{\Omega} \nabla h \cdot \nabla z_{b} .
\end{aligned}
$$


Proof. If we derive the mass equation (1.8) with respect to $x_{i}$ and multiply it by $h \partial_{i} \log h$, when we compute the sum over $i$ and integrate on $\Omega$ (see Ref. 1 ), we get:

$$
\begin{aligned}
\frac{1}{2} \frac{d}{d t} \int_{\Omega} h|\nabla \log h|^{2}+\int_{\Omega} h \nabla \operatorname{div} u \cdot \nabla \log h & \\
& +\int_{\Omega} h \nabla u: \nabla \log h \otimes \nabla \log h=0 .
\end{aligned}
$$

This relation will be used in the following.

We multiply the momentum equation $(1.9)$ by $(\nu / 2) \nabla \log h$ :

$$
\begin{aligned}
\frac{\nu}{2} \int_{\Omega}\left(\partial_{t} u+(u \cdot \nabla) u\right) \cdot \nabla h+\frac{\nu^{2}}{2} \int_{\Omega} D(u) & :\left(\nabla \nabla h-\frac{\nabla h \otimes \nabla h}{h}\right) \\
+ & \frac{\nu}{2 F r^{2}} \int_{\Omega}|\nabla h|^{2}=-\frac{\nu}{2 F r^{2}} \int_{\Omega} \nabla z_{b} \cdot \nabla h .
\end{aligned}
$$

We simplify this expression using the following relations:

$$
\begin{gathered}
\int_{\Omega} h \nabla u: \nabla \log h \otimes \nabla \log h=\int_{\Omega} D(u): \frac{\nabla h \otimes \nabla h}{h}, \\
\int_{\Omega} D(u): \nabla \nabla h+\int_{\Omega} \nabla \operatorname{div} u \cdot \nabla h=0,
\end{gathered}
$$

and add Eq. (3.6) multiplied by $\nu^{2} / 2$. We get:

$$
\begin{aligned}
& \frac{\nu^{2}}{4} \frac{d}{d t} \int_{\Omega} h|\nabla \log h|^{2}+\frac{\nu}{2 F r^{2}} \int_{\Omega}|\nabla h|^{2} \\
& \quad=-\frac{\nu}{2} \int_{\Omega}\left(\partial_{t} u+(u \cdot \nabla) u\right) \cdot \nabla h-\frac{\nu}{2 F r^{2}} \int_{\Omega} \nabla z_{b} \cdot \nabla h \\
& \quad=-\frac{\nu}{2} \frac{d}{d t} \int_{\Omega} u \cdot \nabla h+\frac{\nu}{2} \int_{\Omega} h \nabla u:{ }^{t} \nabla u-\frac{\nu}{2 F r^{2}} \int_{\Omega} \nabla z_{b} \cdot \nabla h
\end{aligned}
$$

which ends the proof of Lemma 3.2.

Proof. We come back to the proof of Proposition 3.2.

Eq. (3.5) gives us:

$$
\begin{aligned}
\frac{1}{2} \frac{d}{d t} \int_{\Omega} h|u+\nu \nabla \log h|^{2} & +\frac{\nu}{F r^{2}} \int_{\Omega}|\nabla h|^{2} \\
& =\frac{1}{2} \frac{d}{d t} \int_{\Omega} h|u|^{2}+\nu \int_{\Omega} h \nabla u:{ }^{t} \nabla u-\frac{\nu}{F r^{2}} \int_{\Omega} \nabla h \cdot \nabla z_{b} .
\end{aligned}
$$


We add to this equality the energy inequality (3.1) multiplied by 2 :

$$
\begin{aligned}
\frac{1}{2} \frac{d}{d t} \int_{\Omega} h|u+\nu \nabla \log h|^{2} & +\frac{1}{F r^{2}} \frac{d}{d t} \int_{\Omega}\left|z_{b}+h\right|^{2}+\frac{2 A}{k+2} \frac{d}{d t} \int_{\Omega} h|u|^{k+2} \\
+\frac{1}{2} \frac{d}{d t} \int_{\Omega} h|u|^{2} & +\frac{\nu}{F r^{2}} \int_{\Omega}\left|\nabla\left(h+z_{b}\right)\right|^{2}+\frac{\nu}{2} \int_{\Omega} h\left|\nabla u+{ }^{t} \nabla u\right|^{2} \\
& +\frac{1-2 k}{4} A \nu \int_{\Omega} h\left|\nabla u+{ }^{t} \nabla u\right|^{2}|u|^{k} \leq \nu \int_{\Omega} h \nabla u:{ }^{t} \nabla u,
\end{aligned}
$$

which proves the proposition.

We then know that our system is dissipative. In addition, we can give a priori estimates:

Corollary 3.1. If $\left(h, u, z_{b}\right)$ is solution of the model (1.8)-(1.10), then, thanks to Proposition 3.2, we have:

$$
\begin{array}{ll}
\|\sqrt{h} u\|_{L^{\infty}\left(0, T ;\left(L^{2}(\Omega)\right)^{2}\right)} \leq c \in \mathbb{R}_{+}, \quad & \|\nabla \sqrt{h}\|_{L^{\infty}\left(0, T ;\left(L^{2}(\Omega)\right)^{2}\right)} \leq c, \\
\left\|z_{b}+h\right\|_{L^{\infty}\left(0, T ; L^{2}(\Omega)\right)} \leq c, & \left\|\sqrt{h}|u|^{(k+2) / 2}\right\|_{L^{\infty}\left(0, T ;\left(L^{2}(\Omega)\right)^{2}\right)} \leq c, \\
\left\|\nabla\left(h+z_{b}\right)\right\|_{L^{2}\left(0, T ;\left(L^{2}(\Omega)\right)^{2}\right)} \leq c, & \|\sqrt{h} \nabla u\|_{L^{2}\left(0, T ;\left(L^{2}(\Omega)\right)^{2}\right)} \leq c, \\
\left\|\sqrt{h} D(u)|u|^{k / 2}\right\|_{L^{2}\left(0, T ;\left(L^{2}(\Omega)\right)^{2}\right)} \leq c . &
\end{array}
$$

\section{Convergence theorem.}

This section is devoted to the proof of Theorem 2.1, in four steps. Thanks to the previous estimates, we show the convergence of the different terms that compose the equation.

\subsection{First step: Convergence of the sequences $\left(\sqrt{h_{n}}\right)_{n \geq 1},\left(h_{n}\right)_{n \geq 1}$ and $\left(z_{b_{n}}\right)_{n \geq 1}$.}

First, we give the spaces in which $\left(\sqrt{h_{n}}\right)_{n}$ is bounded.

If we integrate the mass equation, we directly get $\left(\sqrt{h_{n}}\right)_{n}$ in $L^{\infty}\left(0, T ; L^{2}(\Omega)\right)$. Corollary 3.1 gives us $\|\nabla \sqrt{h}\|_{L^{\infty}\left(0, T ;\left(L^{2}(\Omega)\right)^{2}\right)} \leq c$, so we obtain:

$$
\left(\sqrt{h_{n}}\right)_{n} \text { is bounded in } L^{\infty}\left(0, T ; H^{1}(\Omega)\right) .
$$

Moreover, thanks to the mass equation again, we have the following equality:

$$
\partial_{t} \sqrt{h_{n}}=\frac{1}{2} \sqrt{h_{n}} \operatorname{div} u_{n}-\operatorname{div}\left(\sqrt{h_{n}} u_{n}\right),
$$

which gives that $\left(\partial_{t} \sqrt{h_{n}}\right)_{n}$ is bounded in $L^{2}\left(0, T ; H^{-1}(\Omega)\right)$.

Applying Aubin-Simon lemma, we can extract a subsequence, still denoted $\left(h_{n}\right)_{n \geq 1}$, such that $\sqrt{h_{n}}$ strongly converges to $\sqrt{h}$ in $\mathcal{C}^{0}\left(0, T ; L^{2}(\Omega)\right)$.

Let us study now the subsequence $\left(h_{n}\right)_{n}$. According to the property (4.1) and Sobolev embeddings, we know that, for all finite $p,\left(\sqrt{h_{n}}\right)_{n}$ is bounded in 
$L^{\infty}\left(0, T ; L^{p}(\Omega)\right)$. In the following, we will suppose $p \geq 4$ in order to simplify our expressions and ensure that $\left(h_{n}\right)_{n}$ is in $L^{\infty}\left(0, T ; L^{2}(\Omega)\right)$.

The equality $\nabla h_{n}=2 \sqrt{h_{n}} \nabla \sqrt{h_{n}}$ enables us to bound the sequence $\left(\nabla h_{n}\right)_{n}$ in $L^{\infty}\left(0, T ;\left(L^{2 p /(2+p)}(\Omega)\right)^{2}\right)$ and consequently the sequence $\left(h_{n}\right)_{n}$ is bounded in $L^{\infty}\left(0, T ; W^{1,2 p /(2+p)}(\Omega)\right)$.

Moreover, we have some properties on the time derivative of $h_{n}$; actually the mass equation reads: $\partial_{t} h_{n}=-\operatorname{div}\left(h_{n} u_{n}\right)$. Splitting the product $h_{n} u_{n}$ into $h_{n} u_{n}=\sqrt{h_{n}} \sqrt{h_{n}} u_{n}$, we get $\left(h_{n} u_{n}\right)_{n}$ in $L^{\infty}\left(0, T ;\left(L^{2 p /(2+p)}(\Omega)\right)^{2}\right)$ and $\left(\partial_{t} h_{n}\right)_{n}$ in $L^{\infty}\left(0, T ; W^{-1,2 p /(2+p)}(\Omega)\right)$.

Thanks to Aubin-Simon lemma again, we find:

$$
h_{n} \rightarrow h \text { in } \mathcal{C}^{0}\left(0, T ; L^{2 p /(2+p)}(\Omega)\right)
$$

Last, we consider the bottom term $\left(z_{b_{n}}\right)_{n}$ : with Corollary 3.1 and the bound on $\left(\sqrt{h_{n}}\right)_{n}$ in $L^{\infty}\left(0, T ; L^{p}(\Omega)\right)$, we know that the sequence $\left(\nabla z_{b_{n}}\right)_{n}$ is bounded in $L^{2}\left(0, T ;\left(L^{2 p /(2+p)}(\Omega)\right)^{2}\right)$, which gives

$$
\left(z_{b_{n}}\right)_{n} \text { is bounded in } L^{\infty}\left(0, T ; W^{1,2 p /(2+p)}(\Omega)\right) .
$$

For the time derivative of $z_{b_{n}}$, we restart from Eq. (1.10). We have just shown that $\left(\Delta z_{b_{n}}\right)_{n}$ is in $L^{\infty}\left(0, T ; W^{-1,2 p /(2+p)}(\Omega)\right)$. Let us come to the divergence term:

$$
h_{n}\left|u_{n}\right|^{k} u_{n}=h_{n}^{(1-k) / 2}\left(h_{n}^{1 / 2}\left|u_{n}\right|\right)^{k} h_{n}^{1 / 2} u_{n}
$$

where

- $\left(h_{n}^{(1-k) / 2}\right)_{n}$ is bounded in $L^{\infty}\left(0, T ; L^{p /(1-k)}(\Omega)\right)$,

- $\left(\left(h_{n}^{1 / 2}\left|u_{n}\right|\right)^{k}\right)_{n}$ is bounded in $L^{\infty}\left(0, T ; L^{2 / k}(\Omega)\right)$,

- $\left(h_{n}^{1 / 2} u_{n}\right)_{n}$ is bounded in $L^{\infty}\left(0, T ;\left(L^{2}(\Omega)\right)^{2}\right)$,

that is to say $\left(h_{n}\left|u_{n}\right|^{k} u_{n}\right)_{n}$ is bounded in $L^{\infty}\left(0, T ;\left(L^{2 p /(2-2 k+k p+p)}(\Omega)\right)^{2}\right)$. As $0<k<1 / 2$ and we supposed $p \geq 4$, it leads us to: $\left(h_{n}\left|u_{n}\right|^{k} u_{n}\right)_{n}$ is bounded in $L^{\infty}\left(0, T ; L^{4 p /(2+3 p)}(\Omega)\right)$.

Since in our case $4 p /(2+3 p) \leq 2 p /(2+p)$, we obtain:

$$
\left(\partial_{t} z_{b_{n}}\right)_{n} \text { is bounded in } L^{\infty}\left(0, T ; W^{-1,4 p /(2+3 p)}(\Omega)\right) .
$$

As we have the relations $W^{1,2 p /(2+p)}(\Omega) \subset \subset L^{2 p /(2+p)}(\Omega) \subset W^{-1,4 p /(2+3 p)}(\Omega)$, with Aubin-Simon lemma we are able to assert that $z_{b_{n}}$ strongly converges to $z_{b}$ in $L^{2}\left(0, T ; L^{2 p /(2+p)}(\Omega)\right)$. Note that we could also prove the continuity in time with a weaker result in space. 


\subsection{Second step: Convergence of the water discharge}

\section{$\left(\boldsymbol{q}_{n}\right)_{n \geq 1}=\left(h_{n} u_{n}\right)_{n \geq 1}$.}

In the previous part, we proved that the sequence $\left(h_{n} u_{n}\right)_{n}$ is bounded in $L^{\infty}\left(0, T ;\left(L^{2 p /(2+p)}(\Omega)\right)^{2}\right)$ where $p$ is an integer greater that 4 . Writing the gradient as follow:

$$
\nabla\left(h_{n} u_{n}\right)=2 \sqrt{h_{n}} u_{n} \nabla \sqrt{h_{n}}+\sqrt{h_{n}} \sqrt{h_{n}} \nabla u_{n},
$$

since the first term is in $L^{\infty}\left(0, T ; L^{1}(\Omega)\right)$ and the second one belongs to $L^{2}\left(0, T ; L^{2 p /(2+p)}(\Omega)\right)$, we have:

$$
\left(h_{n} u_{n}\right)_{n} \text { bounded in } L^{2}\left(0, T ; W^{1,1}(\Omega)\right) .
$$

Moreover, the momentum equation (1.9) enables us to write the time derivative of the water discharge:

$$
\partial_{t}\left(h_{n} u_{n}\right)=-\operatorname{div}\left(h_{n} u_{n} \otimes u_{n}\right)-\frac{1}{F r^{2}} h_{n} \nabla\left(h_{n}+z_{b_{n}}\right)+\nu \operatorname{div}\left(h_{n} D\left(u_{n}\right)\right) .
$$

We then study each term:

- $\operatorname{div}\left(h_{n} u_{n} \otimes u_{n}\right)=\operatorname{div}\left(\sqrt{h_{n}} u_{n} \otimes \sqrt{h_{n}} u_{n}\right)$ which is in $L^{\infty}\left(0, T ; W^{-1,1}(\Omega)\right)$,

- as $h_{n}$ is in $L^{\infty}\left(0, T ; W^{1,2 p /(2+p)}(\Omega)\right)$, it is also in $L^{\infty}\left(0, T ; L^{p}(\Omega)\right)$ and we can write the following relation:

$h_{n} \nabla\left(h_{n}+z_{b_{n}}\right)$ is in $L^{2}\left(0, T ; L^{2 p /(2+p)}(\Omega)\right) \subset L^{2}\left(0, T ; W^{-1,2 p /(2+p)}(\Omega)\right)$,

- remark that

$$
\begin{aligned}
h_{k} \nabla u_{k} & =\nabla\left(h_{k} u_{k}\right)-u_{k} \otimes \nabla h_{k} \\
& =\nabla\left(\sqrt{h_{k}} \sqrt{h_{k}} u_{k}\right)-2 \sqrt{h_{k}} u_{k} \nabla \sqrt{h_{k}} ;
\end{aligned}
$$

we know that the first term is in $L^{\infty}\left(0, T ; W^{-1,2 p /(2+p)}(\Omega)\right)$ and the second one in $L^{\infty}\left(0, T ; L^{1}(\Omega)\right)$.

So we have $h_{n} D\left(u_{n}\right)$ bounded in $L^{2}\left(0, T ; W^{-1,2 p /(2+p)}(\Omega)\right)$.

Finally, note that these three terms are included in $L^{2}\left(0, T ; W^{-2,2 p /(2+p)}(\Omega)\right)$, which means that $\partial_{t}\left(h_{n} u_{n}\right)$ is also in this space for all $n \geq 1$.

Then, applying Aubin-Simon lemma, we obtain:

$$
\left(h_{n} u_{n}\right)_{n} \text { strongly converges to } q \text { in } L^{2}\left(0, T ; L^{2 p /(2+p)}(\Omega)\right) .
$$

\subsection{Third step: Convergence of $\left(\sqrt{h_{n}} u_{n}\right)_{n \geq 1}$.}

The product $\sqrt{h_{n}} u_{n}$ is nothing else than the ratio $q_{n} / \sqrt{h_{n}}$. For this term, we also want to prove a strong convergence. Compared with ${ }^{15}$, the bound on $\sqrt{h} u^{(k+2) / 2}$ simplifies the computation.

Before studying the convergence, let us develop some properties of the limit water 
discharge. We know that $\left(q_{n} / \sqrt{h_{n}}\right)_{n}$ is bounded in $L^{\infty}\left(0, T ; L^{2}(\Omega)\right)$; consequently Fatou lemma reads:

$$
\int_{\Omega} \liminf \frac{q_{n}^{2}}{h_{n}} \leq \liminf \int_{\Omega} \frac{q_{n}^{2}}{h_{n}}<+\infty .
$$

In particular, $q(t, x)$ is equal to zero for almost every $x$ where $h(t, x)$ vanishes. Then we can define the limit velocity taking $u(t, x)=q(t, x) / h(t, x)$ if $h(t, x) \neq 0$ or else $u(t, x)=0$. So we have a link between the limits $q(t, x)=h(t, x) u(t, x)$ and:

$$
\int_{\Omega} \frac{q^{2}}{h}=\int_{\Omega} h|u|^{2}<+\infty
$$

Moreover, we can use Fatou lemma again to write

$$
\int_{\Omega} h|u|^{k+2} \leq \int_{\Omega} \liminf h_{n}\left|u_{n}\right|^{k+2} \leq \liminf \int_{\Omega} h_{n}\left|u_{n}\right|^{k+2},
$$

which gives $\sqrt{h}|u|^{(k+2) / 2}$ in $L^{2}\left(0, T ; L^{2}(\Omega)\right)$.

As $\left(q_{n}\right)_{n}$ and $\left(h_{n}\right)_{n}$ converge almost everywhere, the sequence of $\sqrt{h_{n}} u_{n}=q_{n} / \sqrt{h_{n}}$ converges almost everywhere to $\sqrt{h} u=q / \sqrt{h}$ when $h$ does not vanish. Moreover, for all $M$ positive, $\left(\sqrt{h_{n}} u_{n} \mathbf{1}_{\left|u_{n}\right| \leq M}\right)_{n}$ converges almost everywhere to $\sqrt{h} u \mathbf{1}_{\mid u \leq M}$ (still assuming that $h$ does not vanish). If $h$ vanishes, we can write $\sqrt{h_{n}} u_{n} \mathbf{1}_{\left|u_{n}\right| \leq M} \leq$ $M \sqrt{h_{n}}$ and then have convergence towards zero. Then, almost everywhere, we obtain the convergence of $\left(\sqrt{h_{n}} u_{n} \mathbf{1}_{\left|u_{n}\right| \leq M}\right)_{n}$.

Finally, let us consider the following norm:

$$
\begin{aligned}
\int_{\Omega}\left|\sqrt{h_{k}} u_{k}-\sqrt{h} u\right|^{2} \\
\leq \int_{\Omega}\left(\left|\sqrt{h_{k}} u_{k} \mathbf{1}_{\left|u_{k}\right| \leq M}-\sqrt{h} u \mathbf{1}_{|u| \leq M}\right|+\left|\sqrt{h_{k}} u_{k} \mathbf{1}_{\left|u_{k}\right|>M}\right|\right. \\
\left.+\left|\sqrt{h} u \mathbf{1}_{|u|>M}\right|\right)^{2} \\
\leq 3 \int_{\Omega}\left|\sqrt{h_{k}} u_{k} \mathbf{1}_{\left|u_{k}\right| \leq M}-\sqrt{h} u \mathbf{1}_{|u| \leq M}\right|^{2}+3 \int_{\Omega}\left|\sqrt{h_{k}} u_{k} \mathbf{1}_{\left|u_{k}\right|>M}\right|^{2} \\
+3 \int_{\Omega}\left|\sqrt{h} u \mathbf{1}_{|u|>M}\right|^{2} .
\end{aligned}
$$

Since $\left(\sqrt{h_{n}}\right)_{n}$ is in $L^{\infty}\left(0, T ; L^{p}(\Omega)\right),\left(\sqrt{h_{n}} u_{n} \mathbf{1}_{\left|u_{n}\right| \leq M}\right)_{n}$ is bounded in this space. So, as we have seen previously, the first integral tends to zero. Let us study the other two terms:

$$
\begin{gathered}
\int_{\Omega}\left|\sqrt{h_{n}} u_{n} \mathbf{1}_{\left|u_{n}\right|>M}\right|^{2} \leq \frac{1}{M^{k}} \int_{\Omega} h_{n}\left|u_{n}\right|^{k+2} \leq \frac{c}{M^{k}}, \\
\int_{\Omega}\left|\sqrt{H} u \mathbf{1}_{|u|>M}\right|^{2} \leq \frac{1}{M^{k}} \int_{\Omega} h|u|^{k+2} \leq \frac{c^{\prime}}{M^{k}},
\end{gathered}
$$

for all $M>0$. When $M$ tends to the infinity, our two integrals tend to zero. Then

$$
\left(\sqrt{h_{n}} u_{n}\right)_{n} \text { strongly converges to } \sqrt{h} u \text { in } L^{2}\left(0, T ; L^{2}(\Omega)\right) \text {. }
$$




\subsection{Fourth step: Convergence of the diffusion terms, the pressure and the solid transport flux.}

Concerning the diffusion term, $\left(\nabla\left(h_{n} u_{n}\right)\right)_{n}$ converges to $\nabla(h u)$ in the sense of the distributions, in $\left(\mathcal{D}^{\prime}((0, T) \times \Omega)\right)^{4}$. Since the sequence $\left(\nabla \sqrt{h_{n}}\right)_{n}$ weakly converges in $L^{2}\left(0, T ;\left(L^{2}(\Omega)\right)^{2}\right)$ and $\left(\sqrt{h_{n}} u_{n}\right)_{n}$ strongly converges in this space, then $\left(u_{n} \otimes \nabla h_{n}\right)_{n}$ weakly converges in $L^{1}\left(0, T ;\left(L^{1}(\Omega)\right)^{4}\right)$. So, using the relation (4.3) to write the product $h_{n} \nabla u_{n}$, we have $\left(h_{n} \nabla u_{n}\right)_{n}$ that converges to $h \nabla u$ in $\left(\mathcal{D}^{\prime}((0, T) \times \Omega)\right)^{4}$. This gives the convergence of the complete diffusion term.

From Corollary 3.1, we know that $\left.\nabla\left(h_{n}+z_{b_{n}}\right)\right)_{n}$ weakly converges to $\nabla(h+$ $b)$ in $L^{2}\left(0, T ;\left(L^{2}(\Omega)\right)^{2}\right)$. In addition, the sequence $\left(h_{n}\right)_{n}$ strongly converges in $\mathcal{C}^{0}\left(0, T ; L^{2 p /(2+p)}(\Omega)\right)$ so the product weakly converges to $h \nabla\left(h+z_{b}\right)$ in $L^{2}\left(0, T ;\left(L^{p /(1+p)}(\Omega)\right)^{2}\right)$.

The last term is the term of solid transport flux: $\left(h_{n}^{(1-k) / 2}\right)_{n}$ strongly converges to $h^{(1-k) / 2}$ in $\mathcal{C}^{0}\left(0, T ; L^{2 /(1-k)}(\Omega)\right)$ and $\left(\sqrt{h_{n}} u_{n}\right)_{n}$ strongly converges to $\sqrt{h} u$ in $L^{2}\left(0, T ;\left(L^{2}(\Omega)\right)^{2}\right)$. Moreover, $\left(h_{n}^{k / 2}\left|u_{n}\right|^{k}\right)_{n}$ strongly converges to $h^{k / 2}|u|^{k}$ in $L^{2 / k}\left(0, T ; L^{2 / k}(\Omega)\right)$. Using Eq. (4.2), we obtain that the sequence $\left(h_{n}\left|u_{n}\right|^{k} u_{n}\right)_{n}$ strongly converges to $h|u|^{k} u$ in the space $L^{2 /(k+1)}\left(0, T ;\left(L^{1}(\Omega)\right)^{2}\right)$.

This ends the proof of Theorem 2.1.

\section{Others sediment discharge choices.}

\subsection{Model coming from those studied above.}

Let us consider the bed-load transport model

$$
\partial_{t} z_{b}+\operatorname{div}(h u)=0 .
$$

This model of sediment has been studied in Ref. 21 but, in this article, the ShallowWater system is taken as in Ref. 17, that is to say the viscous term is a laplacian. Here, we couple Eq. (5.1) with the Shallow-Water system used above, given by (1.8)(1.9). We prove that this model can be studied as an usual Shallow-Water system. Indeed, combining (1.8) and (5.1) we get

$$
\partial_{t}\left(z_{b}-h\right)=0
$$

and, by an integration with respect to $t$, we obtain

$$
z_{b}(t, x)=h(t, x)-z_{b_{0}}(x)+h_{0}(x) .
$$

Setting $b(x)=h_{0}(x)-z_{b_{0}}(x)$, the expression of $z_{b}$ becomes

$$
z_{b}(x, t)=h(x, t)-b(x) .
$$

If we replace $z_{b}$ by this value in (1.9), we get:

$$
\partial_{t}(h u)+\operatorname{div}(h u \otimes u)+\frac{h \nabla(2 h-b(x))}{F r^{2}}-A \operatorname{div}(h D(u))=0 .
$$


Hence, the problem becomes no-coupled, which means we can determine $h$ using Eq. (1.8) and (5.3) and then use the relation (5.2) to deduce the value of $z_{b}$, since $b$ is given. The Shallow-Water system (1.8)-(5.3) is studied in Ref. 5 where the authors proved an existence result, under the assumption $b \geq c>0$ and some assumptions on the regularity.

\subsection{Another viscous sediment transport.}

We propose here another viscous system. More precisely, we consider the ShallowWater system

$$
\begin{aligned}
& \partial_{t} h+\operatorname{div}(h u)=0 \\
& \partial_{t}(h u)+\operatorname{div}(h u \otimes u)+\frac{h \nabla\left(h+z_{b}\right)}{F r^{2}}-\nu \operatorname{div}(h D(u))=0
\end{aligned}
$$

with the bed-load equation

$$
\partial_{t} z_{b}+A \operatorname{div}\left(h\left(1+\log \left(1+|u|^{2}\right) u\right)\right)-\frac{\nu}{2} \Delta z_{b}=0 .
$$

As mentioned in the introduction, we have modified the sediment equation. We deal here with the term $1+\log \left(1+|u|^{2}\right) u$ used in Ref. 15 to obtain a better bound on $h u^{2}$. As for the previous model, multiplying the diffusion term by $1+\log \left(1+|u|^{2}\right) u$ gives some terms which are controllable. We get the existence of dissipative energy for this system.

Lemma 5.1. Let $\left(h, u, z_{b}\right)$ be a smooth solution of (5.4)-(5.6). The following estimate holds

$$
\begin{gathered}
\frac{1}{2} \frac{d}{d t} \int_{\Omega} h|u|^{2}+A \frac{d}{d t} \int_{\Omega} h \frac{1+|u|^{2}}{2} \log \left(1+|u|^{2}\right)+\frac{1}{2 F r^{2}} \frac{d}{d t} \int_{\Omega}\left|z_{b}+h\right|^{2} \\
+\nu(1-3 A) \int_{\Omega} h(D(u): D(u))+\frac{\nu}{2 F r^{2}} \int_{\Omega} \nabla h \cdot \nabla z_{b} \\
+A \nu \int_{\Omega} h(D(u): D(u)) \log \left(1+|u|^{2}\right)+\frac{\nu}{2 F r^{2}} \int_{\Omega}\left|\nabla z_{b}\right|^{2} \leq 0 .
\end{gathered}
$$

Lemma 5.2. Let $\left(h, u, z_{b}\right)$ be a smooth solution of (5.4)-(5.6). We have

$$
\begin{gathered}
\frac{1}{2} \frac{d}{d t} \int_{\Omega} h|u+\nu \log h|^{2}+A \frac{d}{d t} \int_{\Omega} h \frac{1+|u|^{2}}{2} \log \left(1+|u|^{2}\right) \\
+\frac{1}{2 F r^{2}} \frac{d}{d t} \int_{\Omega}\left|z_{b}+h\right|^{2}+A \nu \int_{\Omega} h(D(u): D(u))+\nu \int_{\Omega} h(W(u): W(u)) \\
+A \nu \int_{\Omega} h(D(u): D(u)) \log \left(1+|u|^{2}\right)+\frac{\nu}{F r^{2}} \int_{\Omega}|\nabla h|^{2} \\
+\frac{\nu}{2 F r^{2}} \int_{\Omega}\left|\nabla z_{b}\right|^{2}+\frac{3 \nu}{2 F r^{2}} \int_{\Omega} \nabla h \cdot \nabla z_{b} \leq 4 A \nu \int_{\Omega} h(D(u): D(u))
\end{gathered}
$$

where $W(u)$ is the skew-symmetric part of the gradient: $W(u)=\frac{\nabla u-{ }^{t} \nabla u}{2}$. 
As in section 3, if we sum the two estimates (5.7) and (5.8), we deduce some bounds on $h, u$ and $z_{b}$ with the condition $0<A<1 / 6$. These bounds allow us to prove the stability of the system (5.4)-(5.6).

\section{Numerical experiments}

\subsection{Numerical scheme}

The proposed model can be written under the structure of a 2D hyperbolic system with non-conservative terms plus the diffusion terms:

$$
\partial_{t} W+\operatorname{div}(\mathcal{F}(W))+B_{1}(W) \partial_{x} W+B_{2}(W) \partial_{y} W-\nu \operatorname{div}(\mathcal{D}(W))=0,
$$

where

$$
\begin{aligned}
& W=\left(\begin{array}{c}
h \\
h u_{1} \\
h u_{2} \\
z_{b}
\end{array}\right), \quad \mathcal{F}=\left(F_{1}, F_{2}\right), \text { with } \\
& F_{1}(W)=\left(\begin{array}{c}
h u_{1} \\
h u_{1}^{2}+h^{2} /\left(2 F r^{2}\right) \\
h u_{1} u_{2} \\
A h|u|^{k} u_{1}
\end{array}\right), \quad F_{2}(W)=\left(\begin{array}{c}
h u_{2} \\
h u_{1} u_{2} \\
h u_{2}^{2}+h^{2} /\left(2 F r^{2}\right) \\
A h|u|^{k} u_{2}
\end{array}\right) \\
& B_{1}(W)=\left(\begin{array}{cccc}
0 & 0 & 0 & 0 \\
0 & 0 & 0 & h / F r^{2} \\
0 & 0 & 0 & 0 \\
0 & 0 & 0 & 0
\end{array}\right), \quad B_{2}(W)=\left(\begin{array}{cccc}
0 & 0 & 0 & 0 \\
0 & 0 & 0 & 0 \\
0 & 0 & 0 & h / F r^{2} \\
0 & 0 & 0 & 0
\end{array}\right) \\
& \mathcal{D}(W)=\left(\begin{array}{c}
h D(u) \\
\nabla z_{b} / 2
\end{array}\right) .
\end{aligned}
$$

The discretization of the model has been done with a high order finite volume method for the hyperbolic system and a centered second order discretization of the diffusion terms.

The following notation is considered (see Figure 2): We decompose the spatial domain in a mesh of cells, finite volumes, $V_{i} \subset R^{2}$ for $i=1 . . N V$. The area of the volume $V_{i}$ is denoted by $\left|V_{i}\right|$ and the center of the cell by $\mathrm{x}_{i}$. We consider that $V_{i}$ is a closed polygon and the boundary of $V_{i}$ is defined by the union of the segments $E_{i j}$, where $E_{i j}$ is the common edge between the volumes $V_{i}$ and $V_{j}$. The normal unit vector to $E_{i j}$ pointing towards $V_{j}$ is denoted by $\eta_{i j}$. The length of the segment $E_{i j}$ is $\left|E_{i j}\right|$. The middle point of $E_{i j}$ is $c_{i j}$. By $b_{i j}$ we denote the baricenter of $V_{i j}$, where $V_{i j}$ is the triangle defined by $E_{i j}$ and $\mathrm{x}_{i}$. Its area is denoted by $\left|V_{i j}\right| . K_{i}$ is the set of indexes $j$ such that $V_{j}$ is a neighbor of $V_{i}$.

We use a second order finite volume method for 2D non-conservative hyperbolic

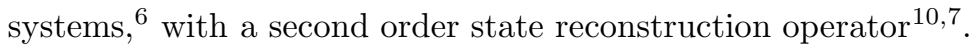




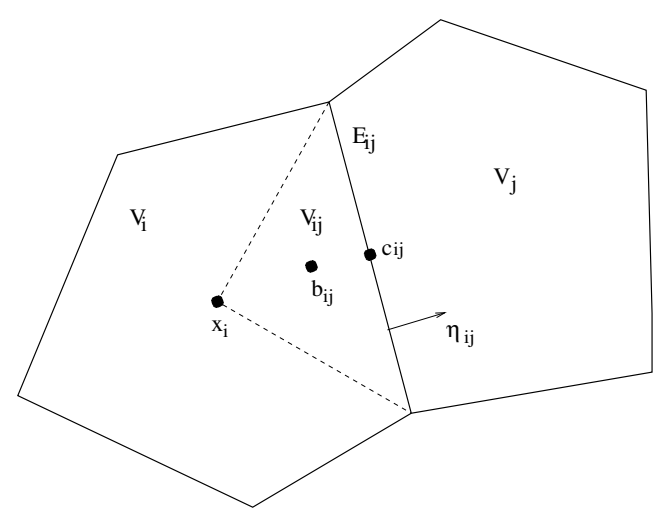

Fig. 2. Finite volume mesh

By $W_{i}(t)$ we denote the average value of $W(x, t)$ over the volume $V_{i}$. And we consider a state reconstruction operator over each volume $P_{i}(\mathrm{x}, t), \mathrm{x} \in V_{i}$, $\left(P_{i}(\mathrm{x}, t) \approx W(\mathrm{x}, t) \forall \mathrm{x} \in V_{i}\right)$; concretely we use a MUSCL second order reconstruction operator ${ }^{7}$. We denote $W_{i j}^{+}(t)=P_{j}\left(c_{i j}, t\right)$ and $W_{i j}^{-}(t)=P_{i}\left(c_{i j}, t\right)$.

We obtain the following numerical scheme,

$$
\begin{gathered}
W_{i}^{\prime}(t)=-\frac{1}{\left|V_{i}\right|}\left[\sum_{j \in K_{i}}\left(\left|E_{i j}\right|\left(G_{i j}-B_{i j}\left(W_{i j}^{+}-W_{i j}^{-}\right)\right)\right)\right. \\
\left.+\sum_{j \in K_{i}}\left|V_{i j}\right|\left(B_{1}\left(P_{i}\left(b_{i j}\right)\right) \partial_{x} P_{i}\left(b_{i j}\right)+B_{2}\left(P_{i}\left(b_{i j}\right)\right) \partial_{y} P_{i}\left(b_{i j}\right)\right)+\sum_{j \in K_{i}}\left|E_{i j}\right| \mathcal{D}_{i j} \eta_{i j}\right],
\end{gathered}
$$

where $G_{i j}=G\left(W_{i j}^{-}(t), W_{i j}^{+}(t), \eta_{i j}\right)$ is a numerical flux function, for example for Roe $\operatorname{method}^{18}$ :

$$
G(U, V, \eta)=\frac{\mathcal{F}_{\eta}(V)+\mathcal{F}_{\eta}(U)}{2}-\frac{1}{2}\left|\mathcal{A}_{\eta}(U, V)\right|(V-U)
$$

where $\mathcal{F}_{\eta}=F_{1} \eta_{1}+F_{2} \eta_{2} \cdot\left|\mathcal{A}_{\eta}(U, V)\right|$ is the absolute value of matrix $\mathcal{A}_{\eta}(U, V)$ and

$$
\mathcal{A}_{\eta}(U, V)=A_{\eta}(U, V)+B_{1}\left(\frac{U+V}{2}\right) \eta_{1}+B_{2}\left(\frac{U+V}{2}\right) \eta_{2},
$$

where $A_{\eta}(U, V)$ verifies

$$
\mathcal{F}_{\eta}(V)-\mathcal{F}_{\eta}(U)=A_{\eta}(U, V)(V-U) .
$$

Moreover,

$$
B_{i j}=\left(B_{1} \eta_{i j, 1}+B_{2} \eta_{i j, 2}\right)\left(\frac{W_{i j}^{+}+W_{i j}^{-}}{2}\right)
$$


By $\mathcal{D}_{i j}$ we denote a second order approximation of $\mathcal{D}\left(W\left(c_{i j}\right)\right)$. The MUSCL operator reconstruction ${ }^{7,10}$ uses a second order approximation of the derivatives of the vector of unknowns, so the same computations can be used to define $\mathcal{D}_{i j}$.

The discretization in time is done with a second order TVD Runge-Kutta $\operatorname{method}^{20}$.

\subsection{Numerical test}

In this subsection we perform a test where we study the evolution of a sand conical dune in a channel. We compare the results for models defined by (1.8)-(1.9) and one of the considered sediment transport models:

(1) Grass model, defined by (1.4).

(2) The first proposed model, defined by (1.10). In what follows we denote it by $\mathcal{M} S 1$.

(3) The last proposed model defined by (5.6). In what follows we denote it by $\mathcal{M} S 2$.

In this section first we study the results obtained with model $\mathcal{M} S 1$, with and without viscosity and for two different values of the constant of interaction between the fluid and the sediment. After we compare with Grass model and model $\mathcal{M} S 2$.

In this test the sediment layer is deformed gradually towards a star shape, expanding along time with a certain angle ${ }^{10,7,12}$.

DE VRIEND ${ }^{8,9}$ determined a formula that relates the solid transport formula of the model with the spread angle.

Consider a given transport equation defined by

$$
\partial_{t} z_{b}+\partial_{x} S_{x}+\partial_{y} S_{y}=0
$$

where the solid transport discharges $S_{x}, S_{y}$ verifies

$$
S_{x}=\frac{u_{1}}{u_{t o t}} S_{t o t}, \quad S_{y}=\frac{u_{2}}{u_{t o t}} S_{t o t},
$$

where $u_{\text {tot }}=|u|$, and $u=\left(u_{1}, u_{2}\right)$. We denote by $\alpha$ the expansion angle of spread. Under the hypothesis of a weak interaction between the fluid and the sediment layer, DE VRIEND ${ }^{8,9}$ deduces that the angle of spread can be approximated by the following formula

$$
\tan \alpha=\frac{3 T_{u} \sqrt{3}}{9 T_{u}-8 T_{h}}
$$

where

$$
T_{u}=\frac{u_{t o t}}{S_{t o t}} \frac{\partial S_{t o t}}{\partial u_{t o t}}-1, \quad T_{h}=\frac{h}{S_{t o t}} \frac{\partial S_{t o t}}{\partial h}-1 .
$$

The proposed model $\mathcal{M} S 1$, defined by (1.8)-(1.10), without viscosity, corresponds to set

$$
S_{t o t}=A h|u|^{k+1}=A h u_{t o t}^{k+1} .
$$




\begin{tabular}{|c|c|c|c|c|c|c|c|}
\hline $\mathrm{k}$ & 0.25 & 1 & 2 & 3 & 4 & 5 & 10 \\
\hline$\alpha$ & $7.22^{\circ}$ & $16.99^{\circ}$ & $21.78^{\circ}$ & $24^{\circ}$ & $25.28^{\circ}$ & $26.11^{\circ}$ & $27.93^{\circ}$ \\
\hline
\end{tabular}

Table 1. Values of $\alpha$ for different values of $k$ for Grass model

So,

$$
\frac{\partial S_{t o t}}{\partial u_{t o t}}=A h(k+1) u_{t o t}^{k}, \quad \frac{\partial S_{t o t}}{\partial h}=A u_{t o t}^{k+1} .
$$

Then,

$$
T_{u}=\frac{A h(k+1) u_{t o t}^{k+1}}{S_{t o t}}-1=k, \quad T_{h}=\frac{A h u_{t o t}^{k+1}}{S_{t o t}}-1=0
$$

Then,

$$
\tan \alpha=\frac{\sqrt{3}}{3}
$$

We obtain that the angle of spread is independent of the definition of the parameter $k ; \alpha=30^{\circ}$ for all values of $k$. For the numerical results presented in this section we have set $k=0.25$.

Model $\mathcal{M} S 2$ correspond to

$$
S_{t o t}=q h u_{t o t}\left(1+\log \left(1+u_{t o t}^{2}\right)\right) .
$$

Applying (6.2), we also obtain for this model $\alpha=30^{\circ}$.

Remark 6.1. Observe that for model $\mathcal{M} S 1$ we obtain that the angle of spread is independent of the value of $k$ because $S_{\text {tot }}$ is not independent of $h$. Otherwise, if $S_{t o t}$ is independent of $h$, we obtain $\partial_{h} S_{t o t}=0$, then $T_{h}=-1$, thus

$$
\tan \alpha=\frac{3 k \sqrt{3}}{9 k \sqrt{3}+8} \text {. }
$$

If we omit in our model the dependency of $h$ we obtain the solid transport formula defined by

$$
S_{t o t}=A|u|^{k+1}
$$

and this definition of $S_{t o t}$ corresponds to the definition obtained for Grass model (1.4). Nevertheless we remark that in our model the dependency of $S_{t o t}$ with respect to $h$ is crucial for the proof of the theorical results.

We present in Table 1 the different values obtained for $\alpha$ in function of different values of $k$ for Grass model. The angle of spread of Grass model converge to $30^{\circ}$ with respect to $k$. That is, the angle of spread predicted for the proposed model (1.8)-(1.10) is the limit angle for Grass model.

The classical value of $k$ used with Grass model is $k=2$ (see for example Ref. 12), corresponding to $\alpha=21.78^{\circ}$. We observe in the numerical results that this angle corresponds to the angle of spread of internal level curves of the sand dune. 
For the definition of the constant of interaction between the fluid and the sediment, $A$, observe that it depends on the porosity of the sediment layer,

$$
A=\xi \bar{A}, \quad \xi=\frac{1}{1-\psi_{0}}
$$

where $\psi_{0}$ is the porosity. In this test we consider $\psi_{0}=0.4$ and two different values of $\bar{A}: \bar{A}=0.001$ and $\bar{A}=0.01$. For $\bar{A}=0.001$ corresponding to a very weak interaction between the fluid and the sediment we simulate until $t=360000 \mathrm{~s}$. For $\bar{A}=0.01$, that can be considered as the limit of a weak interaction, we simulate until $t=36000 \mathrm{~s}$.

We use an explicit finite volume method, then we impose a CFL condition. We set for this test the CFL condition to 0.8 . We use a mesh of 7600 control volumes of edge type (see Figure $3(\mathrm{a})$ ). We impose a discharge $q=(10,0)$ and sediment layer thickness $z_{b}=0.1$ in boundary-line corresponding to $x=0$ and free condition boundary-line corresponding to $x=1000$. At lateral walls we impose sliding condition $q \cdot \eta=0$, if by $\eta$ we denote the outward normal vector.

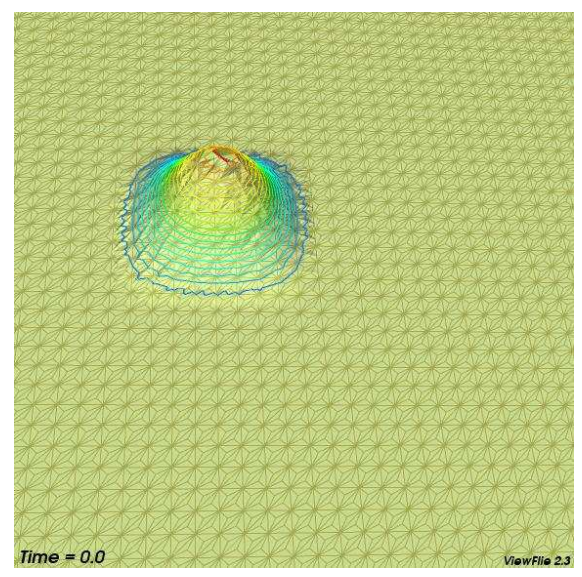

(a) Zoom on the bump in the sediment layer and finite volume mesh

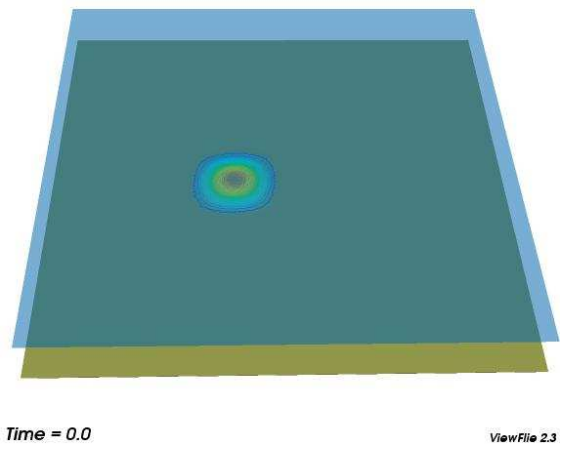

(b) Water surface

Fig. 3. Initial condition.

Initial conditions are (see Figure 3),

$$
h(x, y, 0)=10.1-z_{b}(x, y, 0), q_{x}(x, y, 0)=10, q_{y}(x, y, 0)=0 ;
$$

and the initial sediment layer is a sand dune with a conical form,

$$
z_{b}(x, y, 0)= \begin{cases}0.1+\sin ^{2}\left(\frac{\pi(x-300)}{200}\right) \sin ^{2}\left(\frac{\pi(y-400)}{200}\right) & \text { if } \begin{array}{l}
300 \leq x \leq 500 \\
400 \leq y \leq 600
\end{array} \\
0.1 & \text { other case. }\end{cases}
$$


In Figure 4 we present the evolution of the sand dune for $\bar{A}=0.001$. We superpose the level curves for $t=0, t=180000$ and $t=360000 \mathrm{~s}$. Figure 4(a) corresponds to the model without viscosity and Figure $4(\mathrm{~b})$ to the model with viscosity.

In Figure 5 we present the evolution of the sand dune for $\bar{A}=0.01$. We superpose the level cuves for $t=0, t=18000$ and $t=36000 \mathrm{~s}$. Figure 5 (a) correspond to the model without viscosity and Figure $5(\mathrm{~b})$ to the model with viscosity.

In these figures the continuous black line correspond with an angle of $30^{\circ}$ and the black dashed line with $21.78^{\circ}$.

We observe that for $\bar{A}=0.001$, when the interaction is weaker than for $\bar{A}=0.01$, the analytical solution corresponding to the spread angle of $30^{\circ}$ is better captured. This observation corresponds with the hypothesis under which DE VRIEND deduces formula (6.2); a weak interaction between the fluid and the sediment.

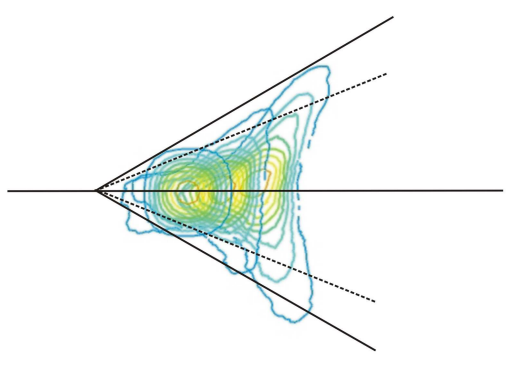

(a) Without viscosity

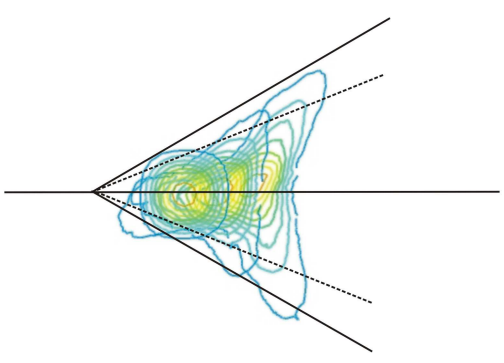

(b) With viscosity

Fig. 4. $\mathcal{M} S 1$. Spread angle, $\bar{A}=0.001$

By comparing the solutions for the model with and without viscosity, we observe that in both cases, $\bar{A}=0.001$ and $\bar{A}=0.01$, the angle of spread is slightly small in the case of the model with viscosity.

As we mentioned previously, Grass model is usually used with $k=2$. In this case we obtain $\alpha=21.78^{\circ}$. We can observe in Figures 4 and 5 that the line corresponding to $\alpha=21.78^{\circ}$ reproduces the angle of spread of an internal level curve (it is also better captured for $\bar{A}=0.001$ than for $\bar{A}=0.01$ ).

The results presented in Figure 6 correspond to $\bar{A}=0.01$, without viscosity. In Figure 6(a) we present the results obtained with Grass model, we observe that effectively the angle of spread approximates the predicted angle of $\alpha=21.78^{\circ}$ 


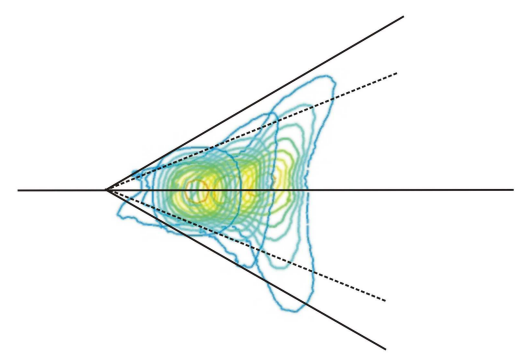

(a) Without viscosity

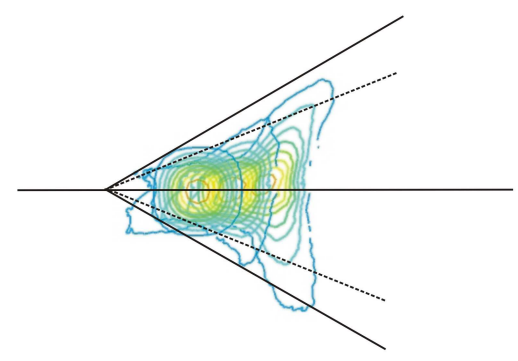

(b) With viscosity

Fig. 5. $\mathcal{M} S 1$. Spread angle, $\bar{A}=0.01$

(discontinous line).

In Figure 6(b) we study the angle of spread of model $\mathcal{M} S 2$. In this case the times of the superposed level curves correspond to $t=0, t=7000$ and $t=14000 \mathrm{~s}$. We also observe that the predicted theorical angle of spread for this model $\left(\alpha=30^{\circ}\right)$ is also well approximated.

Finally, by comparing figures $5(\mathrm{a}), 6(\mathrm{a})$ and $6(\mathrm{~b})$, corresponding to set $\bar{A}=0.01$ in three cases, we can observe that:

(i) Models $\mathcal{M} S 1$ and Grass model, have different angle of spread. But the time evolution obtained with both models are nearly the same (see Figure 7(a)). In both cases, figures $5(\mathrm{a})$ and $6(\mathrm{a})$ the final time is the same.

(ii) Models $\mathcal{M} S 1$ and $\mathcal{M} S 2$, have the same angle of spread. But the time evolution of the sediment layers are different. The final time presented in Figure 5(a) is $t=36000 \mathrm{~s}$. and in Figure 6(b) is $t=14000 \mathrm{~s}$ (see Figure 7(b)). That is, to obtain the same time evolution for the sediment layer the value of $\bar{A}$ must be smaller for $\mathcal{M} S 2$.

\section{Acknowledgments.}

The first author is partially supported by "Réseau EDP-MC, ICTP grants Net 47 and SARIMA". The third author is partially supported by the Spanish Government Research project MTM2006-08075. The computations have been done in the "Laboratorio de Métodos Numéricos de la Universidad de Málaga".

The authors are grateful to D. BRESCH for initiating this work and for the fruitful 


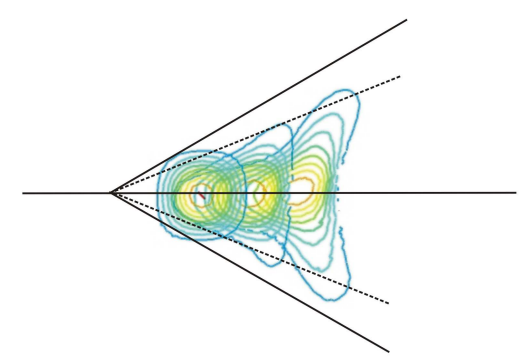

(a) Grass model

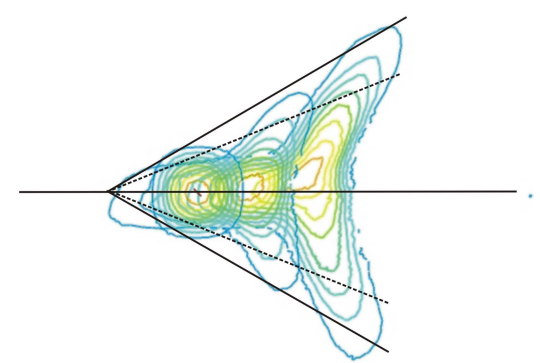

(b) Model $\mathcal{M} S 2$

Fig. 6. Spread angle for Grass model and model $\mathcal{M} S 2$, with $\bar{A}=0.01$

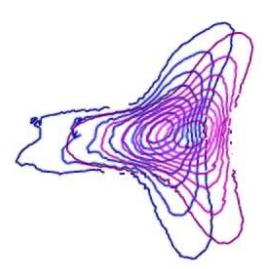

(a) $t=36000 \mathrm{~s}$. for model $\mathcal{M} S 1$ (black lines) and Grass model (magenta lines).

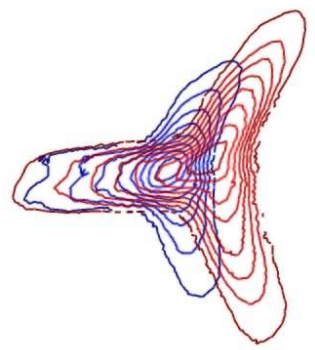

(b) Model $\mathcal{M} S 1$ ( $t=36000$ s., black lines) and model $\mathcal{M} S 2(t=14000 \mathrm{~s}$, red lines)

Fig. 7. Comparison of the level curves

discussions on the subject. The authors would also like to thank the three referees for their comments and suggestions. 


\section{References}

1. D. Bresch and B. Desjardins, Existence of global weak solutions for a $2 \mathrm{D}$ viscous Shallow water equations and convergence to the quasi-geostrophic model, Comm. Math. Phys. 238 (2003) 211-223.

2. D. Bresch and B. Desjardins, On the existence of global weak solutions to the Navier-Stokes equations for viscous compressible and heat conducting fluids, J. Math. Pures Appl. 87 (2007) 57-90.

3. D. Bresch and B. Desjardins, On the construction of approximate solutions for the $2 \mathrm{D}$ viscous shallow water model and for compressible Navier-Stokes models, J. Maths Pures Appl. 86 (2006) 362-368.

4. D. Bresch, B. Desjardins and D. Gérard-Varet, On the compressible NavierStokes equations with density dependent viscosities in bounded domains, J. Math. Pures Appl. 87 (2007) 227-235.

5. D. Bresch, M. Gisclon and C.K. Lin, An example of low Mach (Froude) number effects for compressible flows with nonconstant density (height) limit, M2AN Math. Model. Numer. Anal. 39 (2005) 477-486.

6. M.J. Castro, E.D. Fernández-Nieto, A.M. Ferreiro, J.A. García-Rodríguez and C. PARÉs, High order extensions of Roe schemes for two dimensional nonconservative hyperbolic systems, Preprint (2007).

7. M.J. Castro, E.D. Fernández-Nieto and A.M. Ferreiro, Two dimensional sediment transport models with Shallow Water equations. A second order finite volume approach over non-structured edge type finite volume meshes, Preprint (2007).

8. H.J. De VRIEnd, 2DH Mathematical Modelling of Morphological Evolutions in Shallow Water, Coastal Engineering 11 (1987) 1-27.

9. H.J. De VRIEnd, Analysis of Horizontally Two-Dimensional Morphological Evolutions in Shallow Water, J. Geophys. Res. 92 C4 (1987) 3877-3893.

10. A. M. Ferreiro, Development of post-process technics of hydrodynamics flux, modelization of sediment transport problems and numerical simulation through finite volume technics, PhD Thesis, Seville (2006).

11. A. J. GRAss, Sediment transport by waves and currents, SERC London Cent. Mar. Technol. Report No. FL29 (1981).

12. J. Hudson, Numerical technics for morphodynamic modelling PhD Thesis, University of Whiteknights (2001).

13. J.-L. Lions, Quelques méthodes de résolution des problèmes aux limites non linéaires (Dunod, 1969).

14. P.-L Lions, Mathematical Topics in Fluid Mechanics, Vol. II, Compressible Models Incompressible Models (Clarendon Press. Oxford, 1996).

15. A. Mellet and A. Vasseur, On the barotropic compressible Navier-Stokes equations, Comm. Partial Diff. Eqs 32 (2007) 431-452.

16. E. Meyer-Peter and R. Müller, Formula for bed-load transport, Rep. 2nd Meet. Int. Assoc. Hydraul. Struct. Res., Stockholm (1948) 39-64.

17. P. Orenga, Un théorème d'existence de solutions d'un problème de shallow water, Arch. Rational Mech. Anal. 130 (1995)183-204.

18. C.PARÉs and M.J.CASTRo On the well-balance property of Roe's method for nonconservative hyperbolic systems. Applications to shallow-water systems, ESAIM: M2AN 38(5) (2004) 821-852.

19. C. SAVARY, Transcritical transient flow over mobile beds, boundary conditions treatment in a two-layer shallow-water model, PhD Thesis, Louvain (2007).

20. C.W. SHU and S.OSHER, Efficient implementation of essentially non-oscillatory shock capturing schemes, J. Comp. Phys. 77 (1998) 439-471. 
24 J. D. D. Zabsonré, C. Lucas \& E. Fernández-Nieto

21. B. Toumbou, D. Le Roux and A. Sene, An existence theorem for a 2-D Coupled Sedimentation Shallow-Water Model, C. R. Math. Acad. Sci. Paris 344 Issue 7 (2007) 443-446. 